\title{
Evanescent wave approach to diffractive phenomena in convex billiards with corners
}

\author{
Jan Wiersig, Gabriel G. Carlo \\ Max-Planck-Institut für Physik komplexer Systeme, D-01187 Dresden, Germany
}

(Dated: November 5, 2018)

\begin{abstract}
What we are going to call in this paper "diffractive phenomena" in billiards is far from being deeply understood. These are sorts of singularities that, for example, some kind of corners introduce in the energy eigenfunctions. In this paper we use the well-known scaling quantization procedure to study them. We show how the scaling method can be applied to convex billiards with corners, taking into account the strong diffraction at them and the techniques needed to solve their Helmholtz equation. As an example we study a classically pseudointegrable billiard, the truncated triangle. Then we focus our attention on the spectral behavior. A numerical study of the statistical properties of high-lying energy levels is carried out. It is found that all computed statistical quantities are roughly described by the so-called semi-Poisson statistics, but it is not clear whether the semi-Poisson statistics is the correct one in the semiclassical limit.

PACS numbers: 03.65.Ge, 05.45.Mt
\end{abstract}

\section{INTRODUCTION}

In this work we calculate very high lying eigenvalues of a billiard system using the so-called scaling method [1, 2, 3]. This method has two main advantages, it is formulated on the boundary of the billiard (allowing matrices of order $k$, the wavenumber) and it avoids zeroes searching algorithms. We have solved the Helmholtz equation with Dirichlet boundary conditions on the billiard boundary. A point worth to mention is that any eigenfunction is $C^{\infty}$ at domain points. At straight segments of the boundary, eigenfunctions are reflected as odd functions, so that the result satisfies the Dirichlet condition. Though being also $C^{\infty}$ at the boundary by straight pieces, they are not analytical at a vertex when two segments of the boundary join (with inner angle $\pi / r$, where $r$ is a noninteger number). This feature leads to what we address as diffractive phenomena.

The main idea behind the scaling method is that the trial functions of the variational problem can be parametrised by energy (alternatively, by the wavenumber $k$ ). With this idea in mind these functions are expanded in a suitable scaling basis (plane waves with different propagation directions are one example and these are the elements we use here). Asking the function to be zero at the boundary of the billiard is the same as asking its norm there to be zero as well. Then, eigenvalues (and eigenfunctions) can be obtained by solving a generalised eigenvalue problem that involves the quadratic form associated with the norm of the function on the boundary.

In applying the method to this system, evanescent waves were needed. This is because real plane wave solutions to the Helmholtz equation cannot represent all the features of diffraction. It has been shown [4] that an evanescent plane wave, which oscillates along propagation direction faster than the wavenumber $k$, can be constructed by means of real plane waves. Nevertheless, the corresponding superposition is a singular one, suggesting the direct use of the evanescent functions in the basis. This is the way we have dealt with diffraction.

For simplicity we restrict our considerations to an angle of $3 \pi / 4$. A particularly suitable example to study the effect of this kind of corner on the quantum properties is the polygonal billiard shown in Fig. 1. This billiard is called the truncated triangle. It has been studied in a variety of contexts $[5,6,6,8,6,10,11]$.

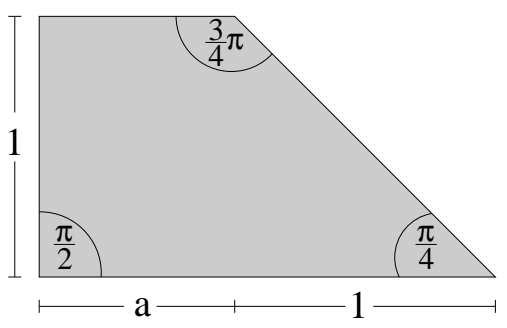

FIG. 1: The truncated triangle.

The truncated triangle belongs to the class of rational polygons. That are polygons where all angles $\alpha_{j}=$ $m_{j} \pi / n_{j}$ between sides are rationally related to $\pi$, where $m_{j}, n_{j}>0$ are relatively prime integers. The free motion inside a rational polygon is integrable if $m_{j}=1$ for all $j$, which is the case for rectangles, the equilateral triangles, the $\pi / 2, \pi / 4, \pi / 4$-triangles and the $\pi / 2, \pi / 3, \pi / 6$ triangles. All other rational polygons are pseudointegrable 5]. Like in integrable systems, the phase space is foliated by two-dimensional invariant surfaces [12, 13]. However, the genus of the surfaces is greater than one due to critical corners with $m_{j}>1$ [5]. In the case of the truncated triangle, the genus is $g=2$. Roughly speaking, the invariant surface is a torus with an additional handle.

Quantum signatures of pseudointegrability can be found in the energy eigenfunctions 14 and in the statistical properties of energy levels [5]. The energy lev-

*Electronic address: jwiersig@mpipks-dresden.mpg.de, carlo@mpipks-dredsdeontmpgredheintegrable systems are correlated in con- 
trast to those of integrable systems which are generically well described by the Poissonian random processes 15]. For example, the nearest-neighbor spacing distribution of pseudointegrable systems generically displays level repulsion [5], resembling the Gaussian orthogonal ensemble (GOE) of random-matrix theory [16] which describes fully chaotic systems with time-reversal symmetry [17]. Significant deviations from GOE have been observed first in Refs. 7, 18]. It has been suggested that the spectral statistics of pseudointegrable systems is another example of critical or intermediate statistics [19, 20]. Critical statistics appear in many condensed matter problems such as in mesoscopic disordered systems at the critical point of the metal-insulator transition [21], in systems with a few interacting electrons [22], and in incommensurate multiwalled carbon nanotubes [23].

In Refs. [19, 20] it has been proposed to use the semiPoisson (SP) statistics as a reference point for critical statistics. The SP statistics is defined by a simple construction: remove every other level from an ordered Poisson sequence [20, 24]. The SP statistics is useful because it provides explicit formulas for a number of statistical quantities which can be compared to the statistical properties of a given system. For several pseudointegrable systems [19, 20, 25, 26, 27, 28, 29] it has been confirmed that the SP statistics indeed describes the shortrange level correlations rather well. Comparing the longrange level correlations is numerically a difficult task because the statistical properties of rational polygons converge extremely slowly as energy is increased [28, 29]. Fortunately, semiclassical periodic-orbit theory allows to compute analytically the long-range level correlations, in terms of the level compressibility $\chi$, of a few special systems, like certain right triangles [28] and the barrier billiard [29]. In the former case $\chi$ differs in general from the $\mathrm{SP}$ result, whereas in the latter case $\chi$ is in agreement with the SP statistics. Nothing is known analytically about the generic case.

Our numerical analysis will show that the truncated triangle is well described by the SP statistics but deviations are not negligible. Our analysis extends that reported in Ref. 7] in many respects: (i) The scaling method allows us to compute more levels, giving a better statistics. (ii) Moreover, high-lying energy levels can be computed. This puts us in a position to study the relevant asymptotic regime. (iii) More statistical quantities are computed. (iv) The numerical results are compared to the SP statistics.

The paper is organized as follows: in Section III we describe the features that are considered when applying the scaling method to the system under investigation. In Section III we present the statistical studies carried out with the data that has been obtained. Finally, Section IV] is devoted to conclusions.

\section{THE SCALING METHOD APPLIED TO THE TRUNCATED TRIANGLE}

We are not going to explain the scaling method here, and we address the reader to the Appendix and the given references for details.

An eigenfunction of a billiard can be constructed as a plane wave superposition. This can include evanescent waves, i.e., plane waves with complex wave vectors. These types of waves should be present in quantum billiards and can be associated with diffractive phenomena. Discontinuities at the boundaries seem to be strongly related to the way evanescent functions must be considered to solve the problem.

Several authors have focused their attention on this issue [2, 4, 30, 31, 32]. For polygonal billiards, in the generic case, theory suggests that there is no real plane wave superposition that can be an eigenfunction 30]. On the other hand, good numerical results using only plane waves could be found [32], but working within the region of low energies. Evanescent waves were studied in detail by Berry 4 in the context of quantum billiards. In this approach, the main reason to do it comes from the idea of constructing them by continuation of an external scattering superposition, containing only real plane waves. In principle, this does not seem to be possible. Anyway, he showed how evanescent waves can be expressed as the singular limit of an angular superposition of real plane waves.

In our system, and working at high energies, we need to consider evanescent waves explicitely, because a real plane wave representation is singular. In the semiclassical limit, the only way to obtain eigenfunctions that include evanescent waves is by considering them in the variational problem [2]. In the remaining part of this section we are going to show some examples of the waves considered and the idea behind their selection.

Plane wave solutions to the Helmholtz equation can be written as

$$
\psi(\mathbf{r})=\exp [i k \cos (\theta+i \alpha) x+i k \sin (\theta+i \alpha) y]
$$

with $\theta$ and $\alpha$ real, using coordinates $\mathbf{r}=(x, y)$ in the plane. We can express this wave in a slightly different fashion by distinguishing the real and imaginary parts in the exponent,

$$
\psi(\mathbf{r})=\exp [i k \cosh (\alpha) \tilde{x}] \exp [-k \sinh (\alpha) \tilde{y}] .
$$

Here, the propagation direction $\tilde{x}=x \cos \theta+y \sin \theta$ implies an angle $\theta$ over the $x$ axis. In this direction the function has a wavelength that is given by $2 \pi /(k \cosh \alpha)<$ $2 \pi / k$. However, in the orthogonal direction $\tilde{y}$ the function is an exponential with coefficient $-k \sinh \alpha$.

An example of these functions applied to our system can be found in Fig. 2] where we show an evanescent wave corresponding to the truncated triangle of parameter value $a=(\sqrt{5}-1) / 2$ (Note that since the absolute size of the system is irrelevant, we measure length scales 
as dimensionless quantities). In this case we chose $k=20$ in order to have a visible wave. For the higher-lying wave numbers $(k \simeq 1000$ or greater) these functions are difficult to see in the domain since they decay very fast. Nevertheless, the slower oscillating functions we have used in the latter energy region can be seen over the boundary.

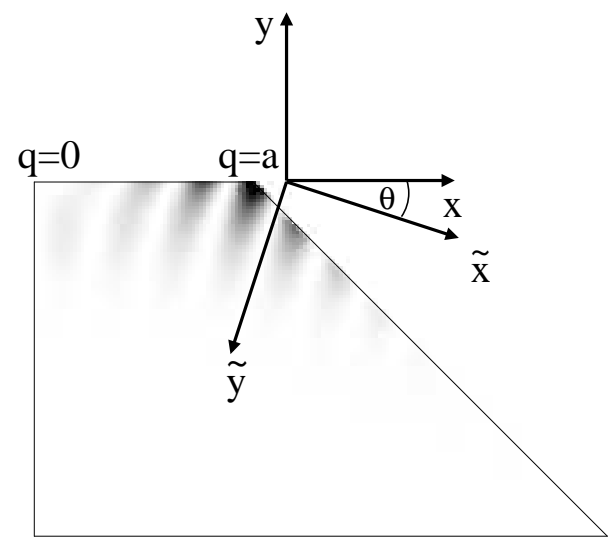

FIG. 2: Evanescent wave for $k=20$ on the truncated triangle domain with parameter value $a=(\sqrt{5}-1) / 2$. The propagation direction $\tilde{x}$ (given by $\sin \theta=-0.3$ in this case) and the corresponding decaying direction $\tilde{y}$ are both shown by the arrows. Also, we point out values 0 and $a$ of the arclength coordinate $q$.

Now that the general ideas related to evanescent waves have been exposed, we are going to explain the way we selected them, and we are also going to show some examples for $k=1000$. The first thing to point out is that diffraction is generated at the critical corner with angle $3 \pi / 4$ that is shown in Fig. 1 So, our sets or families of evanescent waves are centered at this corner. We consider three different kinds of functions, all sharing the previous property, but decaying to one or the other "side" of the corner (in terms of the arclength boundary coordinate $q$ ) in the first place and also an additional one that decays to both sides. We use a family of 12 waves whose propagation direction $\theta$ is slightly smaller than $-\pi / 4$, i.e., around the right hand side of the boundary as seen from Fig. 1 another set of 8 waves with almost horizontal propagation and finally one that goes along an intermediate direction (for this one we took a value of $\sin \theta=-0.3$, as can be seen in Fig. (2). In fact, in order to appreciate their contribution, it is convenient to look at them on the boundary of the billiard. For this reason we show in Fig. 3 three examples of evanescent waves that are good representatives of these three families. As already mentioned, the exponential decay is from the critical corner, which corresponds to $q=a$ in terms of the boundary coordinate. This is the effect of considering an angle slightly different from $-\pi / 4$ angle for the propagation direction. The same happens with the second family whose example is directed almost along the horizontal (left) segment of the boundary. Finally, we show the evanescent wave that decays to both sides of the corner.
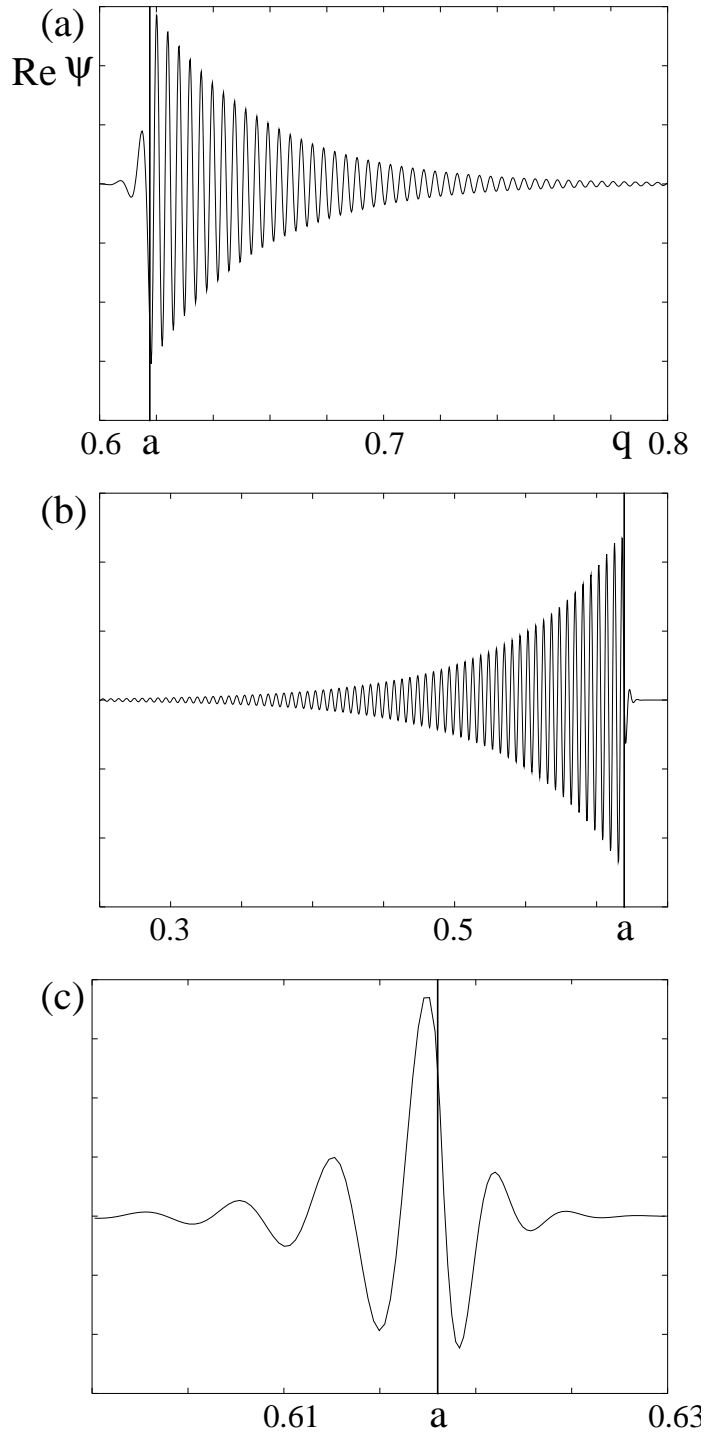

FIG. 3: Three examples of evanescent waves over the billiard boundary for $k=1000$. Vertical axis corresponds to the real part of the evanescent wave in arbitrary units. Horizontal axis corresponds to the boundary arclength coordinate $q$ (dimensionless). In (a) we show a wave decaying to the right (propagation direction given by $\sin \theta=-0.682)$, in (b) one to the left $(\sin \theta=-0.025)$, and in (c) to both sides $(\sin \theta=-0.3)$ from $q=a$, the position of the critical corner.

This approach to the problem has proven to be very efficient. As a matter of fact, studying carefully several billiard eigenfunctions that show the greatest norm (error) over the boundary (without considering evanescent waves in their calculation), we could check that these are the main components of the diffracted field.

Then we apply the symmetries of our system in order to get the right contributions. This is easy to implement by using the symmetry operations of the $C_{2 v}$ group.

We have taken only up to 21 evanescent waves in order to get our results. This is a small number compared to the roughly 1000 real plane waves that are used in the 
highest-lying energy window we have obtained [1]. But they are key to lower the error of the eigenvalues. This shows that, even though the relevance of this contribution goes to zero in the semiclassical limit we need to consider it in order to resolve individual states.

\section{SPECTRAL STATISTICS}

We here examine the spectral statistics of the quantized truncated triangle. We consider the generic case where $a$ is an irrational number. In the nongeneric case of $a$ being rational, the energy spectrum contains a subset of Poisson distributed levels [5]. We focus on the parameter value $a=(\sqrt{5}-1) / 2$, the reciprocal of the golden mean. For other parameter values of $a[(\sqrt{5}-1) / 2+0.2$, $2 / \pi$, and $2 / \pi+0.1]$ similar results have been obtained. We use energy windows of length 20000 in five different regimes starting with level number 853, 32 124, 89607 , 149879 , and 190356 corresponding to the wave number $k=100,600,1000,1300$, and 1500 .

To study the local fluctuations in the level sequence $E_{1} \leq E_{2} \leq E_{3} \leq \ldots$ it is necessary to remove the systematic global energy dependence of the average density. To do so, we "unfold" the spectra in the usual way by setting $\tilde{E}_{n}=\bar{N}\left(E_{n}\right)$; see, e.g., Ref. [33. $\bar{N}(E)$ is the smooth part of the integrated density of states, i.e., the number of levels up to energy $E$. We approximate $\bar{N}(E)$ by the generalized Weyl's law including perimeter and corner corrections 34]

$$
\bar{N}(E)=\frac{A}{4 \pi} E-\frac{L}{4 \pi} \sqrt{E}+C,
$$

where $A=a+1 / 2$ is the area of the billiard, $L=2 a+$ $2+\sqrt{2}$ is the perimeter, and $C=11 / 36$ is the corner correction. The unfolded spectra $\left\{\tilde{E}_{n}\right\}$ have unit mean level spacing and are dimensionless. Henceforth, the tilde will be suppressed.

\section{A. Nearest-neighbor spacing distributions}

The most popular statistical quantity in the field of quantum chaos is the nearest-neighbor spacing distribution. It is defined as the probability density of the spacing $s$ (in units of the mean level spacing) between adjacent levels,

$$
P(s)=\lim _{n \rightarrow \infty} \frac{1}{n} \sum_{i=1}^{n} \delta\left(s-E_{i+1}+E_{i}\right) .
$$

Clearly, the nearest-neighbor spacing distribution is a measure of short-range level correlations. We will compute its integral, the cumulative spacing distribution

$$
I(s)=\int_{0}^{s} P\left(s^{\prime}\right) d s^{\prime} .
$$

For the Poisson statistics $P_{\mathrm{P}}(s)=\exp (-s)$ and $I_{\mathrm{P}}(s)=$ $1-\exp (-s)$, the GOE is well described by the Wigner surmise $P_{\mathrm{W}}(s)=(\pi / 2) s \exp \left(-\pi s^{2} / 4\right)$ and $I_{\mathrm{W}}(s)=1-$ $\exp \left(-\pi s^{2} / 4\right)$, and for the SP statistics [20, 24]

$$
P_{\mathrm{SP}}(s)=4 s e^{-2 s}, I_{\mathrm{SP}}(s)=1-(2 s+1) e^{-2 s} .
$$

At small $s, P_{\mathrm{SP}}(s)$ exhibits a linear increase from zero (level repulsion) similar to the Wigner surmise. At large $s, P_{\mathrm{SP}}(s)$ has an exponential fall-off as the Poisson statistics.

In Fig. 4 we plot the difference between the cumulative spacing distribution to the SP result for the lowest-energy window. Good agreement with the SP statistics can be observed. Figure[5]shows a magnification, containing also the other energy windows. We see increasing deviations from SP for $k=600$ and $k=1000$, and then decreasing deviations for $k=1300$ and $k=1500$. No clear trend to $\mathrm{SP}$ is visible as one goes to higher energies.

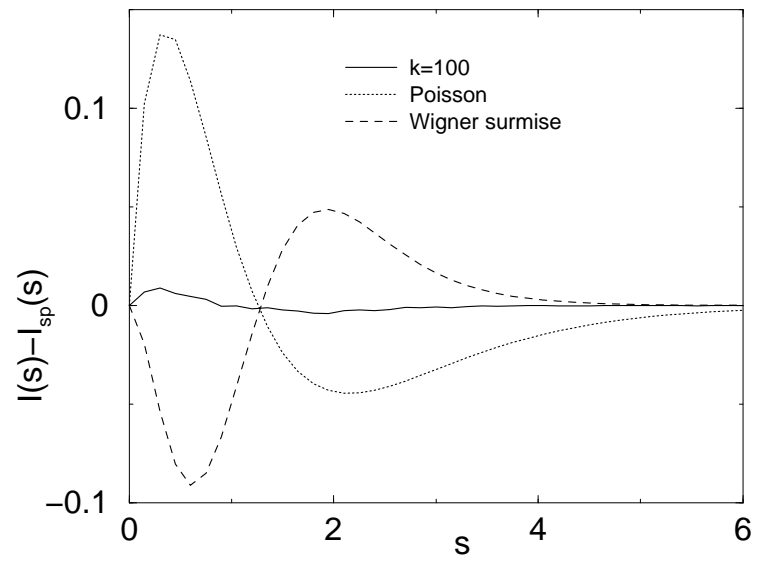

FIG. 4: Difference between the cumulative spacing distribution of the first energy window and the SP result.

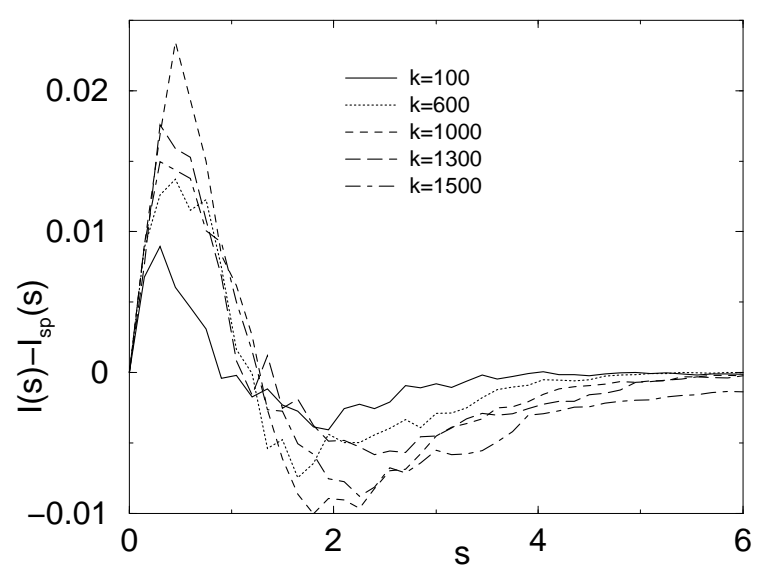

FIG. 5: The cumulative spacing distribution of all energy windows.

Similar small deviations from the SP statistics have been observed at the metal-insulator transition in the 
three-dimensional Anderson model. In that case, an ensemble average over certain boundary conditions removes the deviations considerably 35]. To see whether this is possible also in our case, we consider Neumann boundary conditions on the two boundary segments which do not touch the critical corner; see Fig. 1] In this way we obtain four energy spectra corresponding to Dirichlet/Dirichlet, Dirichlet/Neumann, Neumann/Dirichlet, and Neumann/Neumann-boundary conditions. In contrast to the case of the Anderson model we find that averaging over these boundary conditions does not reduce the deviation from the SP statistics.

\section{B. Next-to-nearest spacing distributions}

We now consider the next-to-nearest spacing distribution (second-neighbor-spacing distribution) and its integral. The SP statistics gives [20]

$$
\begin{aligned}
& P_{\mathrm{SP}}(2, s)=\frac{8}{3} s^{3} e^{-2 s} \\
& I_{\mathrm{SP}}(2, s)=1-\frac{1}{3}\left(4 s^{3}+6 s^{2}+6 s+3\right) e^{-2 s} .
\end{aligned}
$$

Figure 6 shows that the cumulative next-to-nearest spacing distribution is well described by the SP statistics. The maximal deviations from SP are smaller than in the case of the nearest-neighbor spacing distribution

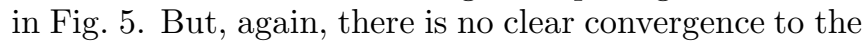
$\mathrm{SP}$ statistics as one goes to higher energies.

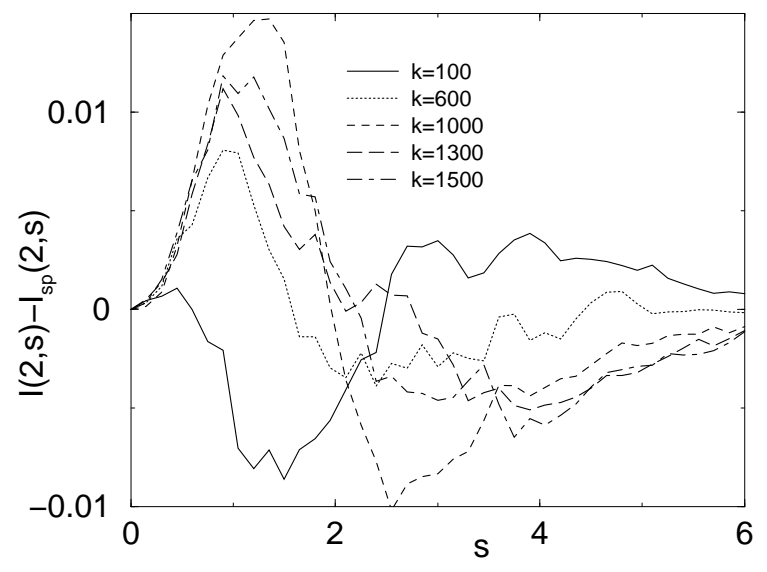

FIG. 6: The cumulative next-to-nearest spacing distribution.

\section{Number variance}

So far we have studied short-range level correlations (nearest and next-to-nearest spacing distributions). Long-range level correlations are conveniently studied with the help of the number variance

$$
\Sigma(L)=\left\langle(n(L, E)-L)^{2}\right\rangle .
$$

$\Sigma(L)$ is the local variance of the number $n(L, E)=$ $N(E+L / 2)-N(E-L / 2)$ of energy levels in the interval $[E-L / 2, E+L / 2]$. For the SP statistics we have [19, 20, 24]

$$
\Sigma_{\mathrm{SP}}(L)=\frac{L}{2}+\frac{1}{8}\left(1-e^{-4 L}\right) .
$$

Figure 7 shows the number variance computed for the five energy windows. Note that the regime is well below the crossover region where $\Sigma(L)$ begins to saturate at a value determined by the shortest periodic orbit [36]. With increasing energy the number variance comes closer to the SP result, without showing a clear stabilization. To estimate the limit curve as $k \rightarrow \infty$ we use the extrapolation procedure described in Ref. [28]: extrapolate point by point (with $L$ fixed) the four highest curves with a fit $A(L)+B(L) / k$. The limit curve, $A(L)$, is shown as dashed curve in Fig. 7 It is closer to the SP result. To measure the difference we concentrate on the slope in the region of large $L$, the so-called level compressibility

$$
\chi=\lim _{L \rightarrow \infty} \frac{\Sigma(L)}{L} .
$$

We get $\chi \approx 0.41$ from the extrapolated curve. This is close to the SP result 28] of $1 / 2$ (1 for Poisson [36] and 0 for GOE [16, 36$]$ ).

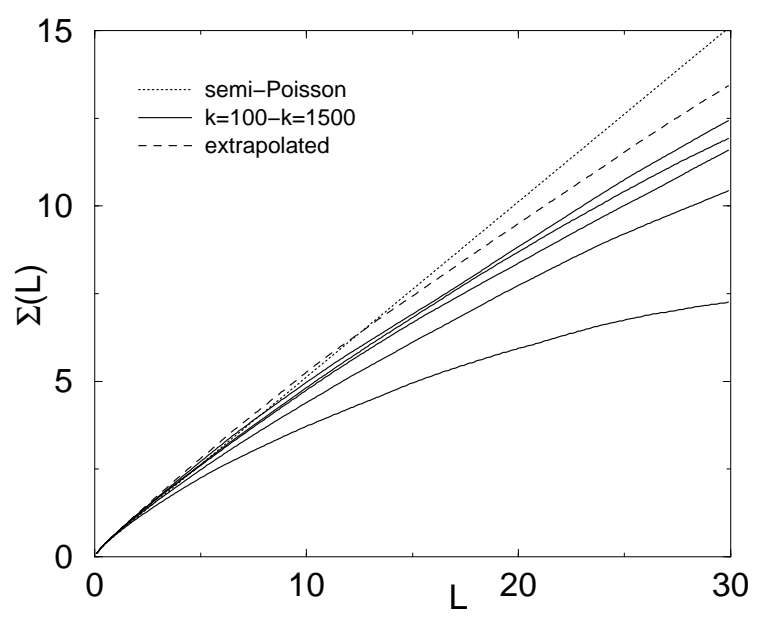

FIG. 7: Number variance $\Sigma(L)$ for $k=100,600,1000,1300$, and 1500 (from below).

\section{The form factor}

Another measure of long-range level correlations is the form factor $K(\tau)$, the Fourier transform of the two-point correlation function. The limit $\tau \rightarrow 0$ is related to the number variance by means of $K(0)=\chi$ (see, e.g., Ref. [28]) with $\chi$ from Eq. [8]. 
The form factor can be approximated numerically by (see, e.g., Ref. [37])

$$
K(\tau ; n)=\frac{1}{n}\left|\sum_{j=l}^{l+n} e^{2 \pi i E_{j} \tau}\right|^{2} .
$$

In our case $n=20000$. Note that $\tau$ is dimensionless. Figure 8 shows $K(\tau ; n)$ averaged over small intervals of size $\Delta \tau=0.006$ in the high-energy regime, i.e., $k=1500$. It is difficult to estimate $K(0)$ from such kinds of noisy data, but it is justified to say that $K(0)$ is below the SP prediction $1 / 2$, in agreement with our former numerical results on the number variance.

A better way to compare the form factor to the SP statistics is introduced in Ref. [28]. Fit $K(\tau ; n)$ to the function

$$
K_{\mathrm{fit}}(\tau)=\frac{c^{2}-2 c+4 \pi^{2} \tau^{2}}{c^{2}+4 \pi^{2} \tau^{2}}
$$

If $c=4$ then function (10) is the SP form factor. We use the quantity $K_{\text {fit }}(0)-1 / 2$ to measure the difference to the SP statistics. Keep in mind that $K_{\text {fit }}(0)$, in general, differs from $K(0 ; n)$ since it depends also on $K(\tau ; n)$ with $\tau>0$. Figure 8 shows the result obtained by fitting Eq. (10) to the smoothed data over the range $0 \leq \tau \leq 3$. We get $K_{\text {fit }}(0) \approx 0.552$. For the lower-energy windows with $k=600, k=1000$, and $k=1300$ we find 0.548 , 0.576 , and 0.56 , respectively.

Again, as for the other spectral quantities described in the previous subsections, we find that the spectral statistics is roughly described by the SP statistics. However, there are small but significant deviations which show no clear trend to zero as the energies are increased.

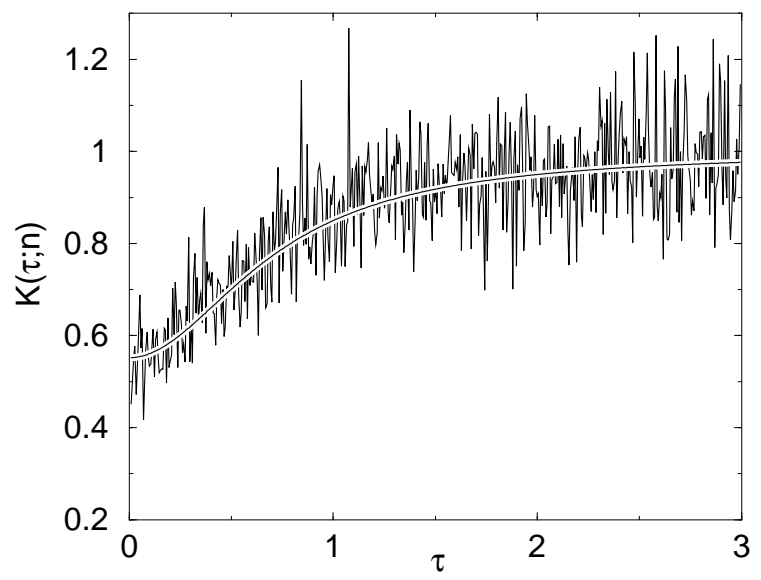

FIG. 8: The form factor 9 for the highest-energy window averaged over small intervals of size $\Delta \tau=0.006$. The smooth curve is the fit 10 with $c=4.464$.

\section{CONCLUSION}

We have shown how the scaling method can be applied to convex billiards with corners. To gain insight into the strong diffractive phenomena that appear in these systems we have studied the truncated triangle. As a result of our investigations of the diffracted field we could identify its main components. These were directly introduced in the function basis in order to obtain the spectral data of this system. Evanescent waves conveniently selected and associated with the centers of diffraction (in our case the two straight segments junction at $3 \pi / 4$ ) have been successfully identified as a very efficient way to deal with these kinds of phenomena. This allowed us to obtain a great number of highly excited eigenvalues.

We have studied the statistical properties of high-lying energy levels in this pseudointegrable billiard. We have found that the nearest-neighbor spacing distributions, next-to-nearest spacing distributions, number variance, and the spectral form factor are roughly described by the semi-Poisson (SP) statistics. Whether the SP statistics is asymptotically the exact statistics cannot be decided.

\section{Acknowledgments}

We would like to thank Eduardo Vergini for his helpful suggestions during the writing of the manuscript. Also for the ideas he gave us regarding the evanescent wave approach to diffraction at corners. Moreover, we would like to thank H. Schomerus and T. Gorin for discussions.

\section{APPENDIX: THE SCALING METHOD}

In this appendix we are going to briefly explain the scaling method [1, 2, 3]. The main point that one should have in mind is that the boundary norm can be written as a function of energy because scaling is used. If $\phi(r)$ satisfies the Helmholtz equation with eigenvalue $k_{0}^{2}$ and we associate with it the scaling function $\phi(k, \mathbf{r})=\phi\left(k \mathbf{r} / k_{0}\right)$ then, these functions satisfy the same equation with eigenvalue $k^{2}$. If we have a billiard defined by a starshaped domain and a $k_{\mu}$ exists such that $\phi_{\mu}\left(k_{\mu}, \mathbf{r}\right)=0$ at the boundary $\mathcal{C}$, then this is a scaling eigenfunction or, equivalently, an eigenfunction of the billiard with Dirichlet boundary conditions.

The boundary norm defined by the expression $f(k)=$ $\oint_{\mathcal{C}} \phi^{2}(k, \mathbf{r}) d l / r_{n}$ can be expanded up to third order around $k_{\mu}$, independently on the exact shape of $\phi$. We remind that $r_{n}=\mathbf{r} \cdot \mathbf{n}$ (where $\mathbf{n}$ is the unit outgoing normal vector to $\mathcal{C}$ ) is always positive for star-shaped domains. Taking into account this result we can evaluate the norm and its derivative in $k$ at value $k_{0}=k_{\mu}+\delta_{\mu}$, obtaining

$$
f\left(k_{0}\right)-\frac{\delta_{\mu}}{2} \frac{d f}{d k}\left(k_{0}\right)+\mathcal{O}\left(\delta_{\mu}^{4}\right)=0 .
$$


This useful expansion, dropping terms of order $\delta_{\mu}^{4}$, turns out to be our quantization condition. Then, all the scaling eigenfunctions with eigenvalues close to $k_{0}$ can be found by solving a generalized eigenvalue problem:

$$
\left[\frac{d F}{d k}\left(k_{0}\right)-\lambda_{\mu} F\left(k_{0}\right)\right] \xi^{\mu}=0 .
$$

In this equation and for numerical calculations, the quadratic form $F$ associated with $f$ can be evaluated in a basis of scaling functions $\psi_{i}(k, \mathbf{r}) ; i=1, \ldots, N$ (like plane waves for instance) by means of

$$
F_{i j}\left(k_{0}\right)=\oint_{\mathcal{C}} \psi_{i}\left(k_{0}, \mathbf{r}\right) \psi_{j}\left(k_{0}, \mathbf{r}\right) d l / r_{n}
$$

The eigenfunctions are $\phi_{\mu}(k, \mathbf{r})=\sum_{i=1}^{N} \xi_{i}^{\mu} \psi_{i}(k, \mathbf{r})$, and the eigenvalues can be found as $k_{\mu}=k_{0}-2 / \lambda_{\mu}$.
[1] E. G. Vergini and M. Saraceno, Phys. Rev. E 52, 2204 (1995).

[2] E. G. Vergini, PhD thesis (Universidad de Buenos Aires, Sept. 1995).

[3] A. Barnett, PhD thesis (Harvard, Sept. 2000).

[4] M. V. Berry, J. Phys. A: Math. Gen. 27, L391 (1994).

[5] P. J. Richens and M. V. Berry, Physica D 2, 495 (1981).

[6] F. S. Henyey and N. Pomphrey, Physica D 6, 78 (1982).

[7] A. Shudo et al., Phys. Rev. E 49, 3748 (1994).

[8] F. Simmel and M. Eckert, Phys. Rev. E 51, 5435 (1995).

[9] F. Simmel and M. Eckert, Physica D 97, 517 (1996).

[10] A. Kudrolli and S. Sridhar, Pramana 48, 459 (1997).

[11] R. Artuso, I. Guarneri, and L. Rebuzzini, Chaos 10, 189 (2000).

[12] A. Hobson, J. Math. Phys. 16, 2210 (1975).

[13] A. Zemlyakov and A. Katok, Math. Notes 18, 760 (1975).

[14] J. Wiersig, Phys. Rev. E 64, 026212 (2001).

[15] M. V. Berry and M. Tabor, Proc. R. Soc. Lond. A 356, 375 (1977).

[16] M. Mehta, Random matrices and the statistical theory of energy levels (Academic Press, New York, 1967).

[17] O. Bohigas, M. Giannoni, and C. Schmit, Phys. Rev. Lett. 52, 1 (1984).

[18] A. Shudo and Y. Shimizu, Phys. Rev. E 47, 54 (1993).

[19] E. B. Bogomolny, U. Gerland, and C. Schmit, Phys. Rev. E 59, R1315 (1999).

[20] E. Bogomolny, U. Gerland, and C. Schmit, Eur. Phys. J. B 19, 121 (2001).

[21] B. Shklovskii et al., Phys. Rev. B 47, 11487 (1993).

[22] X. Waintal, D. Weinmann, and J. Pichard, Eur. Phys. J.
B 7, 451 (1999).

[23] K.-H. Ahn, Y. H. Kim, J. Wiersig, and K. J. Chang, Phys. Rev. Lett. 90, 026601, (2003).

[24] H. Hernández-Saldaña, J. Flores, and T. Seligman, Phys. Rev. E 60, 449 (1999).

[25] B. Grémaud and S. Jain, J. Phys. A 31, L637 (1998).

[26] T. Gorin, J. Phys. A 34, 8281 (2001).

[27] T. Prozen and G. Casati, private communication/preprint (2001).

[28] E. Bogomolny, O. Giraud, and C. Schmit, Commun. Math. Phys. 222, 327 (2001).

[29] J. Wiersig, Phys. Rev. E 65, 046217 (2002).

[30] V. Amar, M. Pauri, and A. Scotti, J. Math. Phys. 32, 2442 (1991).

[31] E. J. Heller, in Chaos and Quantum Physics, Vol. 52 of Les Houches Summer School Lectures, edited by Giannoni et al. (North Holland, Amsterdam, 1991), pp. 547663.

[32] J. Vega, T. Uzer, and J. Ford, Phys. Rev. E 52, 1490 (1995).

[33] F. Haake, Quantum signatures of chaos (Springer, Berlin, 1991).

[34] H. P. Baltes and E. R. Hilf, Spectra of Finite Systems (BI Wissenschaftsverlag, Mannheim, 1976).

[35] D. Braun, G. Montambaux, and M. Pascaud, Phys. Rev. Lett. 81, 1062 (1998).

[36] M. V. Berry, Proc. R. Soc. Lond. A 400, 229 (1985).

[37] J. Marklof, Commun. Math. Phys. 199, 169 (1998). 